\title{
New Insights Into the Role of Aberrant Hippocampal Neurogenesis in Epilepsy
}

\author{
Peng Chen ${ }^{1,2}$, Fuchao $\mathrm{Chen}^{3}$, Yue Wu ${ }^{1}$ and Benhong Zhou ${ }^{1 *}$ \\ ${ }^{1}$ Department of Pharmacy, Renmin Hospital of Wuhan University, Wuhan, China, ${ }^{2}$ Key Laboratory of Combinatorial \\ Biosynthesis and Drug Discovery, Ministry of Education, Wuhan University School of Pharmaceutical Sciences, Wuhan, \\ China, ${ }^{3}$ Sinopharm Dongfeng General Hospital, Hubei University of Medicine, Shiyan, China
}

Data accumulated over the past four decades have confirmed that adult hippocampal neurogenesis $(\mathrm{HN})$ plays a key role in the wide spectrum of hippocampal pathology. Epilepsy is a disorder of the central nervous system characterized by spontaneous recurrent seizures. Although neurogenesis in persistent germinative zones is altered in the adult rodent models of epilepsy, the effects of seizure-induced neurogenesis in the epileptic brain, in terms of either a pathological or reparative role, are only beginning to be explored. In this review, we described the most recent advances in neurogenesis in epilepsy and outlooked future directions for neural stem cells (NSCs)

OPEN ACCESS

Edited by:

Ayanabha Chakraborti, University of Alabama at Birmingham, United States

Reviewed by:

Sreekanth Puttachary, Oregon State University, United States Victor Rodrigues Santos, Federal University of Minas Gerais, Brazil

*Correspondence: Benhong Zhou benhongzh@whu.edu.cn

Specialty section: This article was submitted to Epilepsy,

a section of the journal

Frontiers in Neurology

Received: 23 June 2021 Accepted: 02 November 2021 Published: 15 December 2021

Citation:

Chen P, Chen F, Wu Y and Zhou B (2021) New Insights Into the Role of Aberrant Hippocampal Neurogenesis in Epilepsy. Front. Neurol. 12:727065.

doi: 10.3389/fneur.2021.727065 and epilepsy-in-a-dish models. We proposed that it may help in refining the underlying molecular mechanisms of epilepsy and improving the therapies and precision medicine for patients with epilepsy.

\section{Keywords: hippocampal neurogenesis, epilepsy, pathology, neural stem cells, therapy}

\section{INTRODUCTION}

Despite being assumed to be non-existent for decades, the occurrence and regulation of hippocampal neurogenesis (HN) in adult mammals has been widely accepted (1). Since various hormonal and environmental regulators are identified, this emerging form of adult brain plasticity is getting wide attention and has been studied (2). It is clear that these positive results further increase the interest in $\mathrm{HN}$ following the (re)confirmation that they existed in the adult human brain, which was paralleled by a drastic debate over the sequencing methods, disease model construction, and a possible reinterpretation of the functional role in the human brain (3).

Adult neurogenesis in the mammalian brain is a process by which functional neurons are generated from the division of neural stem cells (NSCs), which have a high capacity for long-term self-renewal while giving rise to the neurons and glia in the subventricular zone/olfactory bulb (SVZ/OB) system and hippocampal dentate gyrus (DG) from the embryo throughout the lifespan of animals (4). Conclusive evidence has confirmed that such a process in the adult subventricles and hippocampi was first documented in rodents, and then extensively explored in human postmortem brains (5). Specifically, granule neurons derived from adult hippocampal neurogenesis (AHN) in the DG are the first relay station in the information flow entering the hippocampus, and their firing rates are strongly regulated by different types of local interneurons, constituting an effective electrophysiological balance stabilizer. When disrupted by a variety of insults, such as brain injury that include stroke and status epilepticus (SE), there is hyperexcitability of hippocampal neurons and a variety of abnormal behaviors, such as memory deficits and decreased motor skills $(6,7)$. 
The production of neurons decreases with age, possibly due to an alteration of the neurogenic niche in the SVZ, a developing environment, or the limited neurogenic capacity of NSCs in the brain (8). Over time, it may cause a progressive agerelated depletion of the stem pool, although neuron production in the hippocampus can still be increased with the use of glucocorticoids and neurosteroids, or exposure of animals to environmental enrichment comprising physical activity (9). Several experimental studies on rodents have shown that AHN during aging is critically involved in learning, memory, and repair because the number and function of NSCs are reduced, resulting in fewer new neurons (10). Furthermore, the extent of cognition and certain types of brain repair are quantitatively linked to the time dependence of HN rates (11).

Over the years, multiple neurological diseases, such as epileptic seizures, ischemic stroke, Alzheimer's disease, and traumatic brain injury, have been demonstrated to be involved in the hippocampal neurogenic cascade in multiple ways (12). These changes have been primarily identified in epilepsy models and are referred to as aberrant $\mathrm{HN}$, encompassing a wide variety of abnormal symptoms and alterations in the pathogenic cascade and size, spine numbers, morphology, function, and location of newborn granule neurons, which are fundamentally different from those under the stage of normal neurogenesis $(13,14)$. Here, we reviewed the recent findings of aberrant $\mathrm{HN}$ in epilepsy to improve our understanding of this field.

\section{PATHOLOGIES OF AHN IN EPILEPSY}

Adult hippocampal neurogenesis in animals or humans is a multi-step process that includes migration, activation, proliferation, differentiation of local NSCs, and neuronal differentiation; thus, each stage may be more vulnerable to the dysregulation brought about by the pathological environment and external stimuli (15). In the case of seizures, multiple dysfunctional outcomes have been identified in rodent animal studies (Figure 1) (16). These changes mainly include an imbalance in quiescence and activation of adult NSCs, alterations in self-renewal rate of NSCs, decrease in the proliferative capacity of NSCs, neural progenitor cells, or neuroblasts, existence of aberrant integrations-hilar basal dendrites, hilar ectopic migration, mossy fiber sprouting in the DG-generated cells, and aberrant migration of newborn neurons into the dentates $(17,18)$. In particular, the development of an abnormal dendritic tree has been confirmed to be a significant feature of AHN because low synaptic connectivity prevents immature neurons from responding broadly to cortical activity, potentially contributing to an imbalance between excitation and inhibition (19). These abnormalities in the number and morphology of newborn neurons can result in the recruitment of newly generated neurons into functional hippocampal networks, creating recurrent excitatory circuits. Furthermore, excessive activation-coupled astrocytic differentiation of NSCs causes profound changes in the maturation-related phenotypes of neurons in the hippocampal DG and the function of hippocampal circuits (20). Thus, it can be hypothesized that the aberrant neurogenesis in the hippocampus is associated with recurrent seizures in patients with epilepsy. In addition, learning, memory, and psychiatric symptoms that include mood disorders, such as anxiety and depression, are affected by hippocampal neuroendocrine regulation and may become exacerbated. Moreover, AHN in epilepsy is likely to be commonly associated with brain dysfunction, leading to several behavioral comorbidities, such as anxiety and depression with learning and memory deficits $(21,22)$.

At present, among the common neurodegenerative diseases associated with AHN, one of the most studied is epilepsy and psychogenic non-epileptic seizures (23). Although the heterogeneities of the experimental animal models have been reported in the literature for epilepsy, it is universally acknowledged that epileptic seizures are often accompanied by an increasing rate of $\mathrm{HN}$, followed by a steady decline (24). Furthermore, there is an abnormal development of morphological changes and functions in neurons generated in the animal hippocampus $(5,12)$. Remarkably, evidence from a recent study (25) of neurogenesis in the adult primate brain indicates that changes in neuronal long-lasting structures due to plasticity are a prominent feature of seizure-associated abnormal neurogenesis. In addition, the depletion of the NSC pool will be accelerated by the hyperactivation of NSCs caused by epilepsy, followed by a long-lasting decrease in NSC number at the later stages of AHN (26).

With the extensive research and understanding of stem cells, hyperactivation of NSCs has been studied in detail in recent years. Upon stimulation with inflammation and stress from convulsive seizures, quiescent NSCs (qNSCs, a type of slowly dividing cells) in the subgranular zone (SGZ) enter the cell cycle and become proliferative NSCs (27). Once activated after epilepsy, the activated NSCs (aNSCs) have the potential to principally divide asymmetrically to generate another NSC and amplify neural progenitors, which are representative of a pluripotent and highly proliferative state to maintain the NSC pool while expanding the progenitor pool (28). Asymmetric division coincides with cell fate determination, and newborn cells that generate aNSCs can further differentiate into reactive astrocytes through multiple rounds of asymmetric division, thereby contributing to increased astrogliosis (29). In general, AHN is regulated by both intrinsic and extrinsic cellular factors, and the complex regulation of AHN becomes clear when studying the fate of neural stem/progenitor cells (NSPCs) transplanted into ectopic locations in the brain (30). The well-documented neurogenic areas of the adult brain are the SGZ of the DG in the hippocampus, where new granule cells originate from NSPCs, and areas of the brain where astrocytes are neurogenic. These transplant studies indicate that the local environment or neurogenic niche in the epileptic brain is crucial for the development of NSCs and neurons (31). The hippocampal neurogenic niche is composed of a wide array of cell types, such as NSPCs, neuroblasts and their progeny, mature granule cells, astrocytes, GABAergic interneurons, microglia, macrophages, and endothelial cells connecting NSCs and their progeny to the vasculature $(32,33)$. Together, all these elements provide the hippocampus with a finely tuned microenvironment that is permissive for adult neurogenesis. 


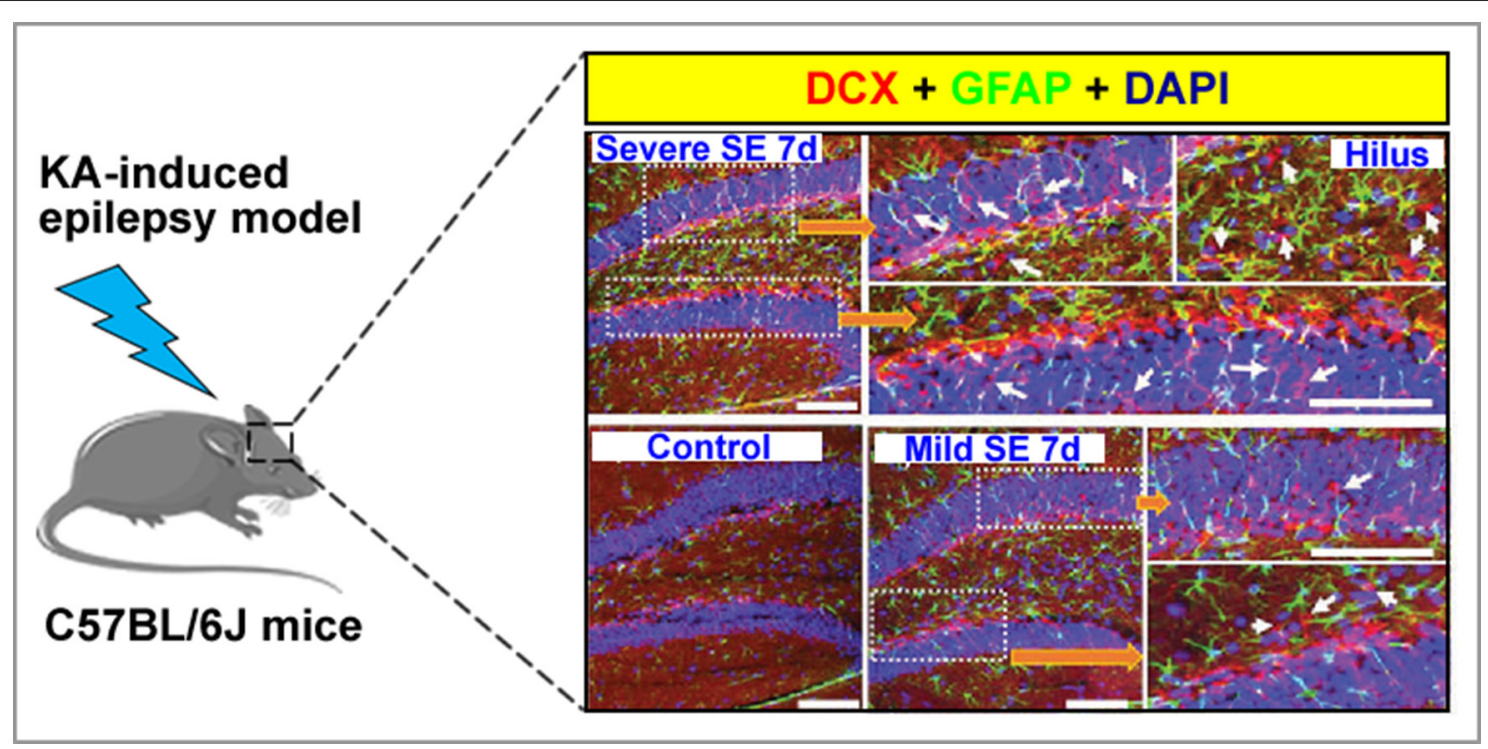

FIGURE 1 | A graph showing normal and aberrant neurogenesis [stained with doublecortin, DCX (red)] in the dentate gyrus of a kainate-induced epilepsy mice model. Arrow indicated that newborn neuron.

Understanding how physiological stimuli may affect neurogenesis through unique neural stem and progenitor cell populations may reveal mechanisms underlying phenotypic outcomes and provide novel therapeutic targets for disease (34). Interestingly, a recent study (35) revealed that significantly more dividing qNSCs and a corresponding increase in the number of surviving new neurons was observed in the hippocampi of kindled vs. sham-kindled rats. These findings are consistent with studies describing increased numbers of dividing qNSCs in the DG of rodents after electroconvulsive seizures or SE induced by chemo-convulsants kainic acid and pilocarpine (36). Thus, we speculated that qNSCs may be a novel target for the treatment of epilepsy and that aberrant neurogenesis could be a cellular mechanism of seizure development and maintenance. Future work employing complete neurogenesis ablation strategies (i.e., targeting resistant qNSCs) would be required to test whether aberrant neurogenesis (through the upregulation of qNSCs) is a cellular mechanism for epilepsy or physiological response to seizures. In the latter case, aberrantly connected young neurons could still provide a cellular substrate for seizure maintenance.

\section{GLIAL FUNCTION IN ABERRANT HN}

Neurogenesis is a common physiological phenomenon that continues throughout life and represents the ability of brain cells to regenerate themselves. In most cases, adult neurogenesis in mammals is thought to be beneficial for maintaining physiological homeostasis in the brain and repairing neurological damage. For example, increasing adult $\mathrm{HN}$ is sufficient to reduce anxiety and depression-like behaviors in mice administered corticosterone (37). Moreover, enhancing optogenetic stimulation of adult-born neurons has been shown to specifically improve olfactory learning and long-term memory (38). Therefore, the role of neurogenesis in regulating emotional and cognitive functions has been recognized. A wide range of scholars have focused on this field, and much research has been conducted (39).

Recent studies have also suggested the beneficial roles of activated microglia in neurogenesis in the adult brain for providing structural, metabolic, and trophic roles for new neurons, phagocytic removal of dead cells, and modulation of the adaptive immune system in the central nervous system (40, 41). In addition, aberrant $\mathrm{HN}$ induced by acute seizures has been thought to be among the crucial players in the generation of spontaneous recurrent seizures and memory impairment (42). Whether microglial activity affects seizure-induced aberrant neurogenesis has only begun to be investigated in recent years.

Specifically, minocycline treatment led to a decreased activation of microglial cells, and the aberrant neurogenesis of the hippocampus after pilocarpine-induced acute seizures was attenuated, while this abnormal neurogenesis was promoted when the microglia were activated with lipopolysaccharide (43). More recently, it has been observed that the convulsive seizure-mediated aberrant neurogenesis was ameliorated by microglia in kainic acid (KA)-induced mice via the activation of toll-like receptor 9 (TLR9), a pattern recognition receptor of the innate immune system that recognizes microbial DNA and triggers inflammatory responses (44). In their study, the selfDNAs released from the damaged cells or neurons were sensed by TLR9 in microglia, which activated the NF-kB signaling pathway, causing aberrant seizure-induced neurogenesis. Another interesting study showed that microglia promoted seizure-induced aberrant neurogenesis through its P2Y12 receptor $(\mathrm{P} 2 \mathrm{Y} 12 \mathrm{R})$ and increased seizure-induced immature 
neuronal projections between the DG and CA3 regions (45). Their results identified microglial P2Y12R as an important regulator of neurogenesis and suggested that targeting it may be a potential method for pro-epileptogenic processes (46). Taken together, these data suggest that microglia are involved in the regulation of the phenotype of acute epileptic seizures and play an important role in the proliferation, survival, and development of NSCs and neuritis during epileptic seizures (45).

It is still uncertain what triggers microglia, and a matter of debate whether microglial activation is beneficial or detrimental to neuronal death and neurogenesis following seizures (47). Therefore, there is no consensus on whether they are neuroprotective or neurotoxic in the human brain associated with epileptic seizures. To clarify the physiological and pathological roles of microglia in brain homeostasis and AHN in epilepsy, investigations on the interaction between the cells and their extracellular environment outside of the adult brain parenchyma are essential for researchers $(39,42)$. Recently, it has also been confirmed that there is a disruption in the structure and function of the blood-brain barrier in the progression of epilepsy, which suggests that cerebrovascular accidents may contribute to microglia-mediated neurogenesis in epilepsy (48). To prevent the development of epilepsy, it is crucial to discover the cellular and molecular mechanisms underlying the synaptic excitatory and inhibitory (E/I) imbalance of AHN during epileptogenesis (49). Furthermore, to prevent the development or progression of epilepsy, it is crucial to reveal the specific cellular and molecular mechanisms underlying synaptic E/I imbalance produced by AHN during epileptogenesis (50). Even if epilepsy has long been considered a synaptopathy and microglia are essential for the development of functional neural circuits, there have been no studies investigating the relationship between microglia and synapses in the AHN of epilepsy.

Astrocytes are also an important cell type in the neurogenic niche and provide a special environment for adult neurogenesis. Similar to microglia, astrocytes serve as important mediators that drive immune responses and promote inflammation. Inflammation is considered one of the greatest causes of neurogenesis, but some cytokines released may have neuroprotective functions. Interleukin (IL)- 6 and IL- $1 \beta$ are the cytokines released from astrocytes and are proposed to act as protectors in the promotion of neuronal differentiation $(51,52)$. A previous study found that stem cell factor restores NPC proliferation in IL-6 knockout mice and that both hippocampus-dependent cognitive functions and the level of adult neurogenesis are gradually attenuated (53). Similar to the results of previous in vivo studies, there was also an improvement in astrocyte-specific IL-6 knockout mice (54). These results provide evidence that astrocytic IL-6, produced under physiological conditions, promotes neurogenesis and supports cognitive function. However, contrary to these findings, overexpression of astrocytic IL-6 decreased neurogenesis and alleviated hippocampus-dependent learning (55).

\section{ROLES OF MICRORNAS IN ABERRANT HN}

The relief of AHN caused by epileptic seizures has become a hot topic of current research, and scholars have put forward many assumptions during this period. In recent years, miRNAs (small non-coding RNAs) have gained significant attention as accumulating evidence has shown that most miRNAs play a crucial role in regulating $\mathrm{AHN}$, and are also deregulated in the cases of seizures or chronic epilepsy (56). Here, an overview of AHN-regulating miRNAs associated with epilepsy was presented based on the analyses performed using EpimiRBase developed by Mooney et al. (57). EpimiRBase is a searchable database (one of the largest manually curated target databases) indexing more than 2,000 miRNA sequences and annotations linked to epilepsy, which were developed to address the rapidly increasing need to track the progress of published literature on miRNAs related to epilepsy-induced aberrant AHN. The recently published EpimiRBase has updated miRNAs from more than 54 publications, and the results showed that 2,183 unique sequences (1,208 upregulated and 975 downregulated) have been found, which mainly consist of three species: humans, mice, and rats (58). Through an integrated analysis of these original miRNAs, they can be categorized into three classes based on their molecular functions: expression analysis, functional, and profiling-biofluid in the brain. Numerous matches have been provided by searchin EpimiRBase against the families of the upregulated and downregulated select miRNAs, which have also been implicated as crucial regulators of AHN, highlighting the crucial role of miRNAs in regulating AHN after SE (59).

The list of miRNAs that regulate AHN continues to grow, and AHN-focused miRNA-based therapy may be a promising strategy for epilepsy, although the complexity by which miRNAs regulate biological processes makes it challenging. Interestingly, several attempts to identify miRNA-based anti-epileptic therapies have been performed, although they have not primarily focused on AHN (56-58). Accumulating evidence suggests that the alterations in miRNA-34a expression may be involved in the pathogenesis of epilepsy (60). Two different rodent epilepsy studies have undertaken miR-34a silencing approaches to rescue seizure-induced apoptosis $(61,62)$. One study found that neuronal apoptosis in CA1 and CA3 was successfully in remission after antagomir-34a administration during SE. However, this was not assessed in the DG; thus, caution should be exercised when extrapolating these findings to AHN (61). Another study also silenced miR-34a using antagomirs, but no neuroprotective effects were observed after a 24 -h post SE induction, indicating a potential time-dependent treatment window (62). From these data, we concluded that antagomir$34 \mathrm{a}$ administration has no beneficial effects on the duration and severity of seizures.

More compelling evidence for the role of miRNA-based regulation in epilepsy implications for therapy comes from several studies on developing possible targets for antagomir-134 anti-epileptogenic effects (63). As mentioned before, miR-134 is known to be involved in regulating synapse formation and dendritic spinogenesis; thus, it may be a key regulator of intrinsic excitability and susceptibility to seizures (64). A significantly greater proportion of pilocarpine-induced SE has been observed in developing rats after pretreatment of mice with antagomir-134 over $24 \mathrm{~h}$ before SE induction (65). In addition, in antagomirtreated mice that developed SE, seizure onset was delayed and the total seizure power was reduced. In another study, the results 


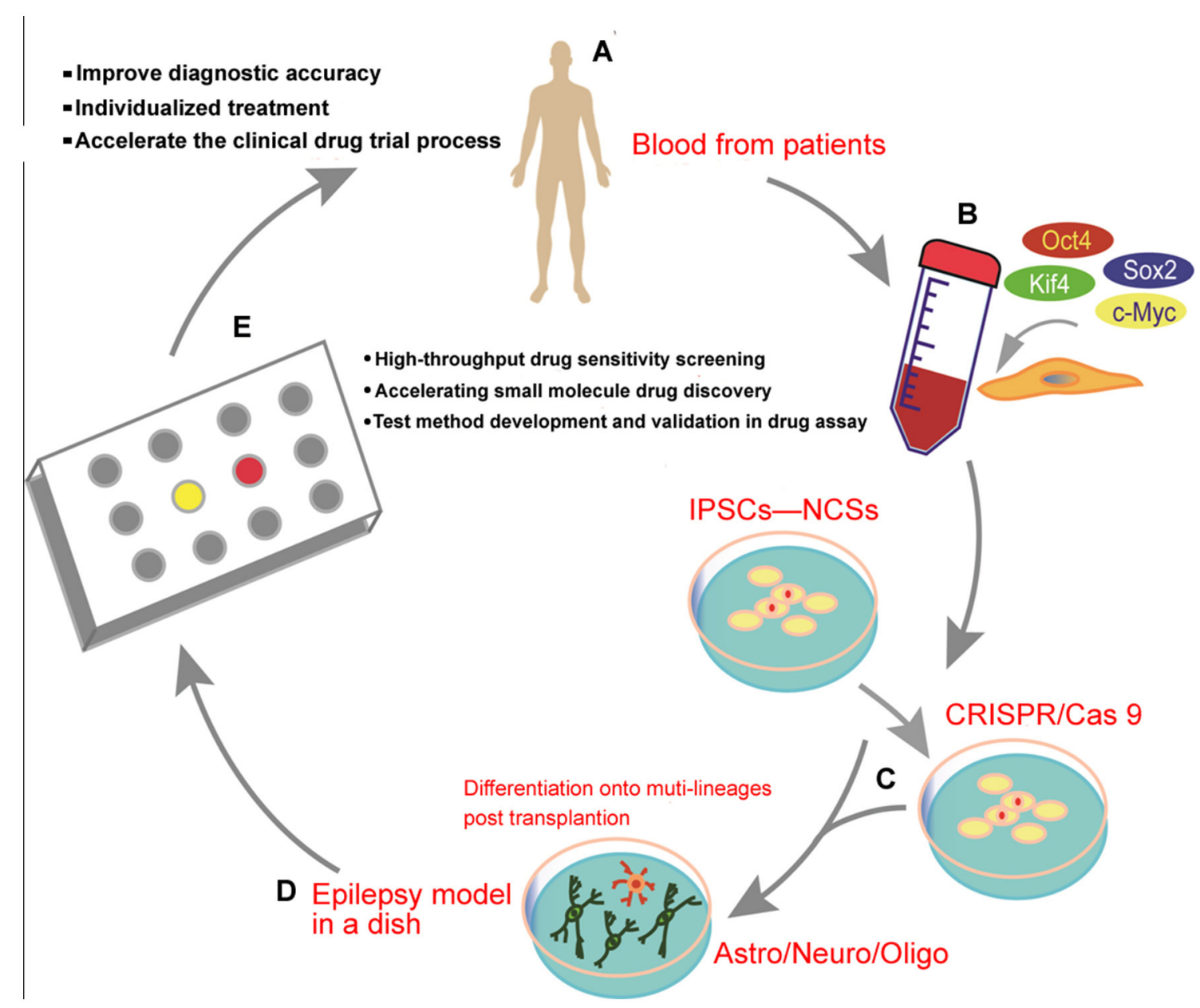

FIGURE 2 | Schematic diagram illustrating the transformation of human-induced pluripotent stem cells (iPSCs) from the clinical trial in a dish to precision medicine for epilepsy: (A) clinical patient recruitment, (B) generation of human iPSCs from the blood of the patients, (C) gene targeting can be effectively achieved using the CRISPR/Cas9 editing system, (D) in vitro generation of human iPSC-derived brain organoids from a mixed population of neurons and glial cells, and (E) drug discovery, individualized therapy, or a clinical trial using the epilepsy-in-a-dish model.

showed that the later occurrence of spontaneous seizures was induced by over $90 \%$ after depletion of miR-134 $1 \mathrm{~h}$ after the onset of SE (66). Furthermore, the results indicated that miR1 antagomir treatment significantly improved the neurological deficits and reduced CA3 pyramidal spine density, hippocampal astrogliosis, and neuronal cell loss, which are the pathological histological forms of temporal lobe epilepsy (TLE). Despite the above studies on miR-34a, the effects on AHN-related pathology were not measured.

\section{ORGANOIDS AND CRISPR/CAS9 TO STUDY GENETIC EPILEPSY SYNDROMES}

A clear understanding of the early stages of epilepsy development is essential for a thorough investigation into new therapeutic strategies and target discovery and development. A large number of studies have shown that the combined application of rodent models in vivo and human-induced pluripotent stem cells (iPSC)-NPS models in vitro is a hot topic for future research on epilepsy $(67,68)$. Although great efforts have been devoted to elucidating many aspects of the environment of the epileptic brain, the understanding of epilepsy development remains elusive due to the significant difference between the human and rodent physiology models (69). The cell field has been transformed by technological advances in recent decades. The establishment and use of an epilepsy model in a dish have already been artificially realized (70). Human cells can be directly harvested for targeted editing of crop genomes using the CRISPR/Cas system, which is now entering a new era of personalized medicine. Moreover, it has been a hot topic in the medical field, where electrophysiological and histological changes in patients with epilepsy can be erased and studied without the need for human nerve tissue, which is available to patients with both surgical and autopsy specimens (71). In the future, we expect that it will be a motivation to expand the applications 
of the combination of in vivo rodent models and in vitro patient-derived iPSC models (Figure 2) to help us understand the different aspects of epilepsy and other neurodevelopmental disorders (72). Furthermore, we hope that more complex iPSCderived epilepsy model systems can be developed and utilized in the screening of drug candidates, probing disease mechanisms, and advancing novel therapies.

\section{CONCLUSION}

Epilepsy is a set of neurological disorders characterized by recurrent seizures and significant comorbidities. The role of NSCs in epilepsy is well-described, but the exact role of AHN in epileptogenesis remains to be elucidated. Moreover, there is comparatively little co-development of therapies and tests based on our current understanding of epilepsy. Given the rapid

\section{REFERENCES}

1. Eid RS, Chaiton JA, Lieblich SE, Bodnar TS, Weinberg J, Galea LAM. Early and late effects of maternal experience on hippocampal neurogenesis, microglia, and the circulating cytokine milieu. Neurobiol Aging. (2019) 78:117. doi: 10.1016/j.neurobiolaging.2019.01.021

2. Toda T, Parylak SL, Linker SB, Gage FH. The role of adult hippocampal neurogenesis in brain health and disease. Mol Psychiatry. (2019) 24:6787. doi: 10.1038/s41380-018-0036-2

3. Gu Y, Janoschka S, Ge S. Neurogenesis and hippocampal plasticity in adult brain. Curr Top Behav Neurosci. (2013) 15:31-48. doi: 10.1007/7854_2012_217

4. Kuhn HG, Toda T, Gage FH. Adult hippocampal neurogenesis: a coming-of-age story. J Neurosci. (2018) 38:1040110. doi: 10.1523/JNEUROSCI.2144-18.2018

5. Niklison-Chirou MV, Agostini M, Amelio I, Melino G. Regulation of adult neurogenesis in mammalian brain. Int J Mol Sci. (2020) 21:486989. doi: 10.3390/ijms21144869

6. Iqbal M, Xiao X-L, Zafar S, Yang B-P, Si K-W, Han H, et al. Forced physical training increases neuronal proliferation and maturation with their integration into normal circuits in pilocarpine induced status epilepticus mice. Neurochem Res. (2019) 44:2590-05. doi: 10.1007/s11064-019-02877-3

7. Xie F, Liu H, Liu Y. Adult Neurogenesis following ischemic stroke and implications for cell-based therapeutic approaches. World Neurosurg. (2020) 138:474-80. doi: 10.1016/j.wneu.2020.02.010

8. Todd K L, Baker KL, Eastman MB, Kolling FW, Trausch AG, Nelson CE, et al. EphA4 regulates neuroblast and astrocyte organization in a neurogenic niche. J Neurosci. (2017) 37:3331-41. doi: 10.1523/JNEUROSCI.3738-16.2017

9. Encinas JM, Sierra A. Neural stem cell deforestation as the main force driving the age-related decline in adult hippocampal neurogenesis. Behav Brain Res. (2012) 227:433-9. doi: 10.1016/j.bbr.2011.10.010

10. Cameron HA, Glover LR. Adult neurogenesis: beyond learning and memory. Annu Rev Psychol. (2015) 66:5381. doi: 10.1146/annurev-psych-010814-015006

11. Alam MJ, Kitamura T, Saitoh Y, Ohkawa N, Kondo T, Inokuchi K. Adult neurogenesis conserves hippocampal memory capacity. J Neurosci. (2018) 38:6854-63. doi: 10.1523/JNEUROSCI.2976-17.2018

12. Cho KO, Lybrand ZR, Ito N, Brulet R, Tafacory F, Zhang L, et al. Aberrant hippocampal neurogenesis contributes to epilepsy and associated cognitive decline. Nat Commun. (2015) 6:6606-18. doi: 10.1038/ncomms7606

13. Basu S, Suh H. Role of hippocampal neurogenesis in alcohol withdrawal seizures. Brain Plast. (2020) 6:27-39. doi: 10.3233/BPL-200114

14. Snyder JS, Soumier A, Brewer M, Pickel J, Cameron HA. Adult hippocampal neurogenesis buffers stress responses and depressive behaviour. Nature. (2011) 476:458-61. doi: 10.1038/nature10287 advances and the increased reliance on biotechnology, the iPSCderived models hold strong promise for generating more relevant human physiological systems for drug testing, elucidating disease mechanisms, and developing new epilepsy therapies by bridging the gap between model systems and patients.

\section{AUTHOR CONTRIBUTIONS}

PC and BZ conceptualized and designed the study, analyzed, and interpreted data. PC wrote the manuscript. PC, FC, and WY designed the figures. $\mathrm{BZ}$ reviewed the manuscript.

\section{FUNDING}

This study was supported by grants from the National Natural Science Foundation of China (31770381).

15. Jessberger S, Parent JM. Epilepsy and adult neurogenesis. Cold Spring Harb Perspect Biol. (2015) 7:a020677-88. doi: 10.1101/cshperspect.a020677

16. Puttachary S, Sharma S, Thippeswamy A, Thippeswamy T. Immediate epileptogenesis: impact on brain in C57BL/6J mouse kainate model. Front Biosci. (2016) 8:390-411. doi: 10.2741/e775

17. Olariu A, Cleaver KM, Cameron HA. Decreased neurogenesis in aged rats results from loss of granule cell precursors without lengthening of the cell cycle. J Comp Neurol. (2007) 501:659-67. doi: 10.1002/cne.21268

18. Gonçalves JT, Schafer ST, Gage FH. adult neurogenesis in the hippocampus: from stem cells to behavior. Cell. (2016) 167:897-914. doi: 10.1016/j.cell.2016.10.021

19. Danzer SC. Adult neurogenesis in the development of epilepsy. Epilepsy Curr. (2019) 19:316-20. doi: 10.1177/1535759719868186

20. Brooker SM, Bond AM, Peng CY, Kessler JA. $\beta 1$-integrin restricts astrocytic differentiation of adult hippocampal neural stem cells. Glia. (2016) 64:123551. doi: 10.1002/glia.22996

21. Tripathi S, Verma A, Jha SK. Training on an appetitive trace-conditioning task increases adult hippocampal neurogenesis and the expression of Arc, Erk and CREB proteins in the dorsal hippocampus. Front Cell Neurosci. (2020) 14:89. doi: $10.3389 /$ fncel.2020.00089

22. Hattiangady B, Shetty AK. Implications of decreased hippocampal neurogenesis in chronic temporal lobe epilepsy. Epilepsia. (2008) 49:26-41. doi: 10.1111/j.1528-1167.2008.01635.x

23. Fares J, Bou Diab Z, Nabha S, Fares Y. Neurogenesis in the adult hippocampus: history, regulation, and prospective roles. Int J Neurosci. (2019) 129:598611. doi: 10.1080/00207454.2018.1545771

24. Yuan P, Han W, Xie L, Cheng L, Chen H, Chen J, Jiang L. The implications of hippocampal neurogenesis in adolescent rats after status epilepticus: a novel role of notch signaling pathway in regulating epileptogenesis. Cell Tissue Res. (2020) 380:425-33. doi: 10.1007/s00441-01903146-Z

25. Murphy BL, Hofacer RD, Faulkner CN, Loepke AW, Danzer SC. Abnormalities of granule cell dendritic structure are a prominent feature of the intrahippocampal kainic acid model of epilepsy despite reduced postinjury neurogenesis. Epilepsia. (2012) 53:908-21. doi: 10.1111/j.1528-1167.2012.03463.x

26. Upadhya D, Hattiangady B, Castro OW, Shuai B, Kodali M, Kodali M, et al. Human induced pluripotent stem cell-derived MGE cell grafting after status epilepticus attenuates chronic epilepsy and comorbidities via synaptic integration. Proc Natl Acad Sci USA. (2019) 116:28796. doi: 10.1073/pnas.1814185115

27. Lybrand ZR, Goswami S, Zhu J, Jarzabek V, Merlock N, Aktar M, et al. A critical period of neuronal activity results in aberrant neurogenesis rewiring hippocampal circuitry in a mouse model of epilepsy. Nat Commun. (2021) 12:1423-36. doi: 10.1038/s41467-021-21649-8 
28. Bielefeld P, Durá I, Danielewicz J, Lucassen PJ, Baekelandt V, Abrous DN, et al. Insult-induced aberrant hippocampal neurogenesis: functional consequences and possible therapeutic strategies. Behav Brain Res. (2019) 372:11203241. doi: 10.1016/j.bbr.2019.112032

29. Kempermann G, Gage FH, Aigner L, Song H, Curtis MS, Thuret S, et al. Human adult neurogenesis: evidence and remaining questions. Cell Stem Cell. (2018) 23:25-30. doi: 10.1016/j.stem.2018.04.004

30. Obernier K, Cebrian-Silla A, Thomson M, Parraguez JI, Anderson $\mathrm{R}$, Guinto C, et al. Adult neurogenesis is sustained by symmetric self-renewal and differentiation. Cell Stem Cell. (2018) 22:22134.e8. doi: 10.1016/j.stem.2018.01.003

31. Wilhelmsson U, Faiz M, de Pablo Y, Sjöqvist M, Andersson D, Widestrand A, et al. Astrocytes negatively regulate neurogenesis through the Jagged1-mediated notch pathway. Stem Cells. (2012) 30:2320-9. doi: 10.1002/stem.1196

32. Crowther AJ, Song J. Activity-dependent signaling mechanisms regulating adult hippocampal neural stem cells and their progeny. Neurosci Bull. (2014) 30:542-56. doi: 10.1007/s12264-014-1453-5

33. Ostendorf BN, Flenner E, Flörcken A, Westermann J. Phenotypic characterization of aberrant stem and progenitor cell populations in myelodysplastic syndromes. PLoS ONE. (2018) 13:e0197823. doi: 10.1371/journal.pone.0197823

34. Niemiro GM, Allen JM, Mailing LJ, Khan NA, Holscher HD, Woods JA, et al. Effects of endurance exercise training on inflammatory circulating progenitor cell content in lean and obese adults: exercise and circulating progenitor cells in obesity. J Physiol. (2018) 596:2811-20. doi: 10.1113/JP276023

35. Leibowitz JA, Leibowitz JA, Zhou J, Carney PR, Ormerod BK. Sustained somatostatin gene expression reverses kindling-induced increases in the number of dividing Type-1 neural stem cells in the hippocampi of behaviorally responsive rats. Epilepsy Res. (2019) 150:78-94. doi: 10.1016/j.eplepsyres.2019.01.005

36. Smith ZZ, Benison AM, Bercum FM, Dudek FE, Barth DS. Progression of convulsive and nonconvulsive seizures during epileptogenesis after pilocarpine-induced status epilepticus. J Neurophysiol. (2018) 119:181835. doi: 10.1152/jn.00721.2017

37. Hill AS, Sahay A, Hen R. Increasing adult hippocampal neurogenesis is sufficient to reduce anxiety and depression-like behaviors. Neuropsychopharmacology. (2015) 40:2368-78. doi: 10.1038/npp.2015.85

38. Lepousez G, Nissant A, Bryant AK, Gheusi G, Greer CA, Lledo PM. Olfactory learning promotes input-specific synaptic plasticity in adult-born neurons. Proc Natl Acad Sci USA. (2014) 111:13984-3. doi: 10.1073/pnas.1404991111

39. Yoo S, Blackshaw S. Regulation and function of neurogenesis in the adult mammalian hypothalamus. Prog Neurobiol. (2018) 170:53-66. doi: 10.1016/j.pneurobio.2018.04.001

40. Sandvig I, Augestad IL, Håberg AK, Sandvig A. Neuroplasticity in stroke recovery. The role of microglia in engaging and modifying synapses and networks. Eur J Neurosci. (2018) 47:1414-28. doi: 10.1111/ejn.13959

41. Zellinger C, Seeger N, Hadamitzky M, Fischborn S, Russmann V, Wendt $\mathrm{H}$, et al. Impact of the erythropoietin-derived peptide mimetic Epotris on the histopathological consequences of status epilepticus. Epilepsy Res. (2011) 96:241-9. doi: 10.1016/j.eplepsyres.2011.06.009

42. Brulet R, Zhu J, Aktar M, Hsieh J, Cho KO. Mice with conditional NeuroD1 knockout display reduced aberrant hippocampal neurogenesis but no change in epileptic seizures. Exp Neurol. (2017) 293:190-8. doi: 10.1016/j.expneurol.2017.04.005

43. Wang N, Mi X, Gao B, Gu J, Wang W, Zhang Y, et al. Minocycline inhibits brain inflammation and attenuates spontaneous recurrent seizures following pilocarpine-induced status epilepticus. Neuroscience. (2015) 287:144-56. doi: 10.1016/j.neuroscience.2014.12.021

44. Matin N, Tabatabaie O, Falsaperla R, Lubrano R, Pavone P, Mahmood F, et al. Epilepsy and innate immune system: a possible immunogenic predisposition and related therapeutic implications. Hum Vaccin Immunother. (2015) 11:2021-2030. doi: 10.1080/21645515.2015.1034921

45. Mo M, Eyo UB, Xie M, Peng J, Bosco DB, Umpierre AD, et al. Microglial P2Y12 receptor regulates seizure-induced neurogenesis and immature neuronal projections. J Neurosci. (2019) 39:945364. doi: 10.1523/JNEUROSCI.0487-19.2019
46. Peng J, Liu Y, Umpierre AD, Xie M, Tian DS, Richardson JR, et al. Microglial P2Y12 receptor regulates ventral hippocampal CA1 neuronal excitability and innate fear in mice. Mol Brain. (2019) 12:71-8. doi: 10.1186/s13041-019-0492-x

47. Eyo UB, Murugan M, Wu LJ. Microglia-neuron communication in epilepsy. Glia. (2017) 65:5-18. doi: 10.1002/glia.23006

48. Li YJ, Wang L, Zhang B, Gao F, Yang CM. Glycyrrhizin, an HMGB1 inhibitor, exhibits neuroprotective effects in rats after lithiumpilocarpine-induced status epilepticus. J Pharm Pharmacol. (2019) 71:390-9. doi: 10.1111/jphp.13040

49. Sarnat HB, Flores-Sarnat L. Excitatory/inhibitory synaptic ratios in polymicrogyria and down syndrome help explain epileptogenesis in malformations. Pediatr Neurol. (2021) 116:4154. doi: 10.1016/j.pediatrneurol.2020.11.001

50. Sarnat HB, Yu W, Flores-Sarnat L. Keratan sulfate proteoglycan as an axonal insulating barrier in the forebrain of fetuses with alobar/semi-lobar holoprosencephaly. Clin Neuropathol. (2021) 40:70-86. doi: 10.5414/NP301317

51. Jones ME, Lebonville CL, Paniccia JE, Balentine ME, Reissner KJ, Lysle DT. Hippocampal interleukin-1 mediates stress-enhanced fear learning: a potential role for astrocyte-derived interleukin-1ß. Brain Behav Immun. (2018) 67:355-63. doi: 10.1016/j.bbi.2017.09.016

52. Bowen KK, Dempsey RJ, Vemuganti R. Adult interleukin-6 knockout mice show compromised neurogenesis. Neuroreport. (2011) 22:126-30. doi: 10.1097/WNR.0b013e3283430a44

53. Baier PC, May U, Scheller J, Rose-John S, Schiffelholz T. Impaired hippocampus-dependent and -independent learning in IL-6 deficient mice. Behav Brain Res. (2009) 200:192-6. doi: 10.1016/j.bbr.2009. 01.013

54. Erta M, Giralt M, Esposito FL, Fernandez-Gayol O, Hidalgo J. Astrocytic IL-6 mediates locomotor activity, exploration, anxiety, learning and social behavior. Horm Behav. (2015) 73:64-74. doi: 10.1016/j.yhbeh.2015.06.016

55. Samuelsson AM, Jennische E, Hansson HA, Holmäng A. Prenatal exposure to interleukin-6 results in inflammatory neurodegeneration in hippocampus with $\mathrm{NMDA} / \mathrm{GABA}(\mathrm{A})$ dysregulation and impaired spatial learning. Am J Physiol Regul Integr Comp Physiol. (2006) 290:R1345-56. doi: 10.1152/ajpregu.00268.2005

56. Mellios N, Feldman DA, Sheridan SD, Ip JPK, Kwok S, Amoah SK, et al. MeCP2-regulated miRNAs control early human neurogenesis through differential effects on ERK and AKT signaling. Mol Psychiatry. (2018) 23:1051-65. doi: 10.1038/mp.2017.86

57. Mooney C, Becker BA, Raoof R, Henshall DC. EpimiRBase: a comprehensive database of microRNAepilepsy associations. Bioinformatics. (2016) 32:143643. doi: 10.1093/bioinformatics/btw008

58. Agostini M, Tucci P, Steinert JR, Shalom-Feuerstein R, Rouleau M, Aberdam D, et al. microRNA-34a regulates neurite outgrowth, spinal morphology, and function. Proc Natl Acad Sci USA. (2011) 108:21099104. doi: 10.1073/pnas. 1112063108

59. Wakabayashi T, Hidaka R, Fujimaki S, Asashima M, Kuwabara T. MicroRNAs and epigenetics in adult neurogenesis. Adv Genet. (2014) 86:2744. doi: 10.1016/B978-0-12-800222-3.00002-4

60. Wang J, Zheng Y, Cheng X, Xu F, Zhang P, Zhou X, et al. Inhibition of microRNA-34a suppresses epileptiform discharges through regulating notch signaling and apoptosis in cultured hippocampal neurons. Neurochem Res. (2019) 44:1252-61. doi: 10.1007/s11064-019-02772-x

61. Sano T, Reynolds JP, Jimenez-Mateos EM, Matsushima S, Taki W, Henshall DC. MicroRNA-34a upregulation during seizure-induced neuronal death. Cell Death Dis. (2012) 3:e287. doi: 10.1038/cddis.2012.23

62. Hu K, Xie YY, Zhang C, Ouyang DS, Long HY, Sun DN, et al. MicroRNA expression profile of the hippocampus in a rat model of temporal lobe epilepsy and miR-34a-targeted neuroprotection against hippocampal neurone cell apoptosis post-status epilepticus. BMC Neurosci. (2012) 13:115. doi: 10.1186/1471-2202-13-115

63. Gorter JA, Iyer A, White I, Colzi A, van Vliet EA, Sisodiya S, et al. Hippocampal subregion-specific microRNA expression during epileptogenesis in experimental temporal lobe epilepsy. Neurobiol Dis. (2014) 62:508-20. doi: 10.1016/j.nbd.2013.10.026 
64. Morris G, Reschke CR, Henshall DC. Targeting microRNA-134 for seizure control and disease modification in epilepsy. EBioMedicine. (2019) 45:64654. doi: 10.1016/j.ebiom.2019.07.008

65. Jimenez-Mateos EM, Engel T, Merino-Serrais P, FernaudEspinosa I, Rodriguez-Alvarez N, Reynolds J, et al. Antagomirs targeting microRNA-134 increase hippocampal pyramidal neuron spine volume in vivo and protect against pilocarpine-induced status epilepticus. Brain Struct Funct. (2015) 220:2387-99. doi: 10.1007/s00429-014-0798-5

66. Jimenez-Mateos EM, Engel T, Merino-Serrais P, McKiernan RC, Tanaka K, Mouri G, et al. Silencing microRNA-134 produces neuroprotective and prolonged seizure-suppressive effects. Nat Med. (2012) 18:108794 doi: $10.1038 / \mathrm{nm} .2834$

67. Simkin D, Kiskinis E. Modeling pediatric epilepsy through ipsc-based technologies. Epilepsy Curr. (2018) 18:240-5. doi: 10.5698/1535-7597.18.4.240

68. Sterlini B, Fruscione F, Baldassari S, Benfenati F, Zara F, Corradi A. Progress of induced pluripotent stem cell technologies to understand genetic epilepsy. Int J Mol Sci. (2020) 21:482-500. doi: 10.3390/ijms21020482

69. Bershteyn M, Nowakowski TJ, Pollen AA, Di Lullo E, Nene A, Wynshaw-Boris A, et al. Human iPSC-derived cerebral organoids model cellular features of lissencephaly and reveal prolonged mitosis of outer radial glia. Cell Stem Cell. (2017) 20:435-49.e4 doi: 10.1016/j.stem.2016.12.007

70. Wong M. Epilepsy in a dish: an in vitro model of epileptogenesis. Epilepsy Curr. (2011) 11:153-6. doi: 10.5698/1535-7511-11.5.153

71. Liu J, Gao C, Chen W, Ma W, Li X, Shi Y, et al. CRISPR/Cas9 facilitates investigation of neural circuit disease using human iPSCs: mechanism of epilepsy caused by an SCN1A loss-of-function mutation. Transl Psychiatry. (2016) 6:e703-11. doi: 10.1038/tp.2015.203

72. Tidball AM, Lopez-Santiago LF, Yuan Y, Glenn TW, Margolis JL, Clayton Walker J, et al. Variant-specific changes in persistent or resurgent sodium current in SCN8A-related epilepsy patient-derived neurons. Brain. (2020) 143:3025-40. doi: 10.1093/brain/awaa247

Conflict of Interest: The authors declare that the research was conducted in the absence of any commercial or financial relationships that could be construed as a potential conflict of interest.

Publisher's Note: All claims expressed in this article are solely those of the authors and do not necessarily represent those of their affiliated organizations, or those of the publisher, the editors and the reviewers. Any product that may be evaluated in this article, or claim that may be made by its manufacturer, is not guaranteed or endorsed by the publisher.

Copyright (c) 2021 Chen, Chen, Wu and Zhou. This is an open-access article distributed under the terms of the Creative Commons Attribution License (CC BY). The use, distribution or reproduction in other forums is permitted, provided the original author(s) and the copyright owner(s) are credited and that the original publication in this journal is cited, in accordance with accepted academic practice. No use, distribution or reproduction is permitted which does not comply with these terms. 